\title{
Bacteriological profile of surgical site infection and associated risk factors in obstetrics and Gynecology patient
}

\author{
Nikita Dhote ${ }^{1}$, Neena Nagdeo ${ }^{2, *}$ \\ ${ }^{1}$ MBBS Student, ${ }^{2}$ Professor, Dept. of Microbiology, NKP Salve Institute of Medical Sciences and Research Center, Nagpur, \\ Maharashtra, India
}

*Corresponding Author:

Email: neenagdeo@yahoo.co.in

\begin{abstract}
Surgical site infections (SSI) are the second most common cause of nosocomial infections in obstetrics and gynecology patient. It has been estimated that SSI develops in at least $2 \%$ of hospitalized patients undergoing operative procedure. Knowledge of the most likely causative organisms and antibiotic sensitivity or resistance pattern will be of great help in managing the patients. Patients in the Obstetrics and Gynecology department, who had developed signs and symptoms of SSI were included in this study. Surgical sites were considered to be infected according to the set of clinical criteria recommended by CDC definition 1992. When infection was clinically suspected, the exudate was collected from the depth of the wound using two sterile cotton swabs one for preparing smear and another for culture. All the specimens collected were transported immediately to the laboratory for further processing. The samples collected were processed by conventional method according to CLSI guidelines. A total of 300 cases were examined for SSI and 25 were found to be clinically infected with the rate of incidence of infection $8.33 \%$. The commonest organism was klebsiella, Pseudomonas $61.5 \%$ followed by staphylococcus $15.3 \%$.
\end{abstract}

Keywords: Surgical site infection, Gram negative bacilli, Risk factors.

\section{Introduction}

Surgical Site Infection (SSI) is an infection that occurs within 30 days of an operation, (that may be superficial or deep) with at least one of the following reasons. Firstly, purulent drainage, with or without lab confirmation, secondly, organisms isolated from an aseptically obtained culture of fluid or tissue from the incision site, thirdly, at least one of the following signs or symptoms of infection, pain or tenderness, localized swelling, redness or heat or fever and superficial incision deliberately opened up by the surgeon, unless incision is culture negative and lastly, Diagnosis of SSI by the surgeon or attending physician. ${ }^{1}$ Even in the developed world like United States the reported rate of SSI is $2.6 \%$ for all operations. ${ }^{2}$ SSI continues to be a major source of morbidity even after judicious use of surgical skills, scrubbing up techniques, environmental changes in operating room and the use of preventive antibiotics. Many studies have been conducted to know the surgical site infection rates in India.

These infections are usually caused by exogenous and endogenous micro-organisms that enter the operative wound during the course of the surgery. Staphylococcus aureus is the commonest cause of SSI and recently $\mathrm{Gm}$ negative bacilli are incriminated as the cause of SSI. The present study was planned to know rate of SSI in obstetrics and gynaecology surgeries, the nature of organisms causing SSI in our hospital.

\section{Aims and Objectives}

1. To study the rates of SSI in obstetrics and gynaecology.

2. To study the bacteriological profile of surgical site infection in our institute (NKP SIMS).
3. To analyse the associated risk factors.

\section{Materials and Methods}

A cross sectional study was conducted for period of 6 months. Study was conducted after obtaining approval from ethics committee. 300 patients undergoing major surgeries including caesarean sections were included in this study. Patients' information was collected on standard proforma. Informed consent was taken from every patient.

Inclusion Criteria: Female patients undergoing surgeries in obstetrics and gynaecology department at NKP SIMS.

Exclusion Criteria: Patients undergoing surgeries outside the hospital and presenting with surgical site infection.

Patients admitted in Obstetrics and Gynecology department who had undergone surgery were included in this study. Proper history was collected from each patient. Surgical wound was inspected at the time of first dressing and daily thereafter till discharge of patient and then patient was followed up in clinics till the $30^{\text {th }}$ post-operative day. SSI was detected on the basis of the criteria given in the modified CDC definition, 1992. ${ }^{1}$

All the specimens collected were transported immediately to the laboratory for further processing. The samples collected were processed by conventional method according to CLSI guidelines. ${ }^{3}$ In suspected cases of SSI, two Swabs from surgical site wound were collected. One swab was used for smear preparation and another was inoculated on blood agar and MacConkeys agar. Identification of organisms was done by standard microbiological techniques. ${ }^{3}$ 


\section{Results}

Most of the surgeries carried out were emergency

LSCS followed by LSCS and TAH, TAH with BSO

(Table 1).

Table 1: Types of surgery $(n=300)$

\begin{tabular}{|l|c|c|}
\hline Types of surgery & No. of surgery & \% \\
\hline LSCS & 58 & 19.33 \\
\hline Emergency LSCS & 195 & $65 \%$ \\
\hline Salphingotomy & 02 & $0.66 \%$ \\
\hline TAH & 27 & $9 \%$ \\
\hline TAH with BSO & 18 & $6 \%$ \\
\hline Total & 300 & \\
\hline
\end{tabular}

LSCS - Lower segment caesarian section, TAH- Total Abdominal Hysterectomy

TAH with BSO - Total abdominal Hysterectomy with Bilateral Salphingotomy and Ovorectomy

Table 2: Wound infected (Total wound infected $=\mathbf{2 5}(8.33 \%)$

\begin{tabular}{|l|c|c|c|}
\hline \multicolumn{1}{|c|}{ Type of surgery } & No. of surgery & Infected & $\mathbf{\%}$ \\
\hline LSCS & 58 & 05 & $8.6 \%$ \\
\hline Emergency LSCS & 195 & 10 & $5.12 \%$ \\
\hline TAH & 27 & 8 & $29.62 \%$ \\
\hline TAH with BSO & 18 & 02 & $11.11 \%$ \\
\hline Salpingotomy & 02 & 00 & $00 \%$ \\
\hline Total & 300 & 25 & $8.33 \%$ \\
\hline
\end{tabular}

Out of the above surgery most of the SSI was found in total abdominal hysterectomy cases i.e. $29.62 \%$ followed by TAH with BSO i.e. $11.11 \%$ and then by LSCS $8.6 \%$ and emergency LSCS $5.12 \%$. TAH and TAH with BSO are more susceptible for nosocomial infection due to the long duration of surgery and long stay in hospital by patient after surgery (Table 2).

Table 3: Culture positivity

\begin{tabular}{|l|c|c|c|}
\hline Type of surgery & No. of surgery & Wound infected (\%) & Culture positive (\%) \\
\hline LSCS & 58 & $05(8.6 \%)$ & $02(3.44 \%)$ \\
\hline Emergency LSCS & 195 & $10(5.12 \%)$ & $05(5.26 \%)$ \\
\hline TAH & 27 & $8(29.62 \%)$ & $06(22.22 \%)$ \\
\hline TAH with BSO & 18 & $02(11.11 \%)$ & Nil $(00 \%)$ \\
\hline
\end{tabular}

Surgical site infection was proved by culture positivity as shown in Table 3 .

Table 4: Organisms isolated from SSI $(n=13)$

\begin{tabular}{|l|c|c|}
\hline \multicolumn{1}{|c|}{ Organism } & No. of organism & \% \\
\hline Klebsiella & 04 & $30.76 \%$ \\
\hline Pseudomonas & 04 & $30.76 \%$ \\
\hline Staphylococcus & 02 & $15.38 \%$ \\
\hline Actinobacter & 01 & $7.69 \%$ \\
\hline Non fermenter & 01 & $7.69 \%$ \\
\hline Klebsiella+Pseudomonas (Mixed) & 01 & $7.69 \%$ \\
\hline
\end{tabular}

The most common organism isolated from wound infection is Gram negative Klebsiella and Pseudomonas i.e. $30.76 \%$ followed by Gram positive Staphylococcus

aureus i. e. $15.38 \%$ and then by Actinobacter, Non fermenter and mixed growth of Klebsiella and Pseudomonas $7.69 \%$ each (Table 4).

Table 5: Comparison between SSI rates according to nature of surgery

\begin{tabular}{|l|c|c|c|}
\hline Nature of surgery & Total no. of surgery & Infected & \% \\
\hline Emergency & 202 & 16 & $7.92 \%$ \\
\hline Elective & 98 & 09 & $9.18 \%$ \\
\hline Total & 300 & 25 & $8.33 \%$ \\
\hline
\end{tabular}

Rate of SSI was more in elective surgeries (9.18\%) as compared to Emergency surgeries (Table 5). 
Table 6: Associated risk factor

\begin{tabular}{|l|c|c|c|}
\hline \multicolumn{1}{|c|}{ Factors } & No. of patient & Infected & \% \\
\hline PROM & 39 & 06 & $15.38 \%$ \\
\hline Mild Anaemia & 88 & 9 & $10.22 \%$ \\
\hline Moderate Anaemia & 84 & 06 & $7.14 \%$ \\
\hline Severe Anaemia & 12 & 03 & $25 \%$ \\
\hline Diabetics & 02 & 01 & $50 \%$ \\
\hline
\end{tabular}

PROM-Premature rupture of membrane

Table 6 shows that $15.38 \%$ Patients of PROM, $25 \%$ patients of anaemia and $50 \%$ patients of Diabetes developed SSI.

\section{Discussion}

The present study was done on the patients who underwent surgery in obstetrics and gynecology department of Lata Mangeshkar Hospital. Total number of cases included in the study were 300 out of which clinically infected cases were 25 with infection rate of $8.33 \%$ which was well with the range of infection rate in other studies.

In India incidence of surgical site infection varies from 10-25\%. At Addis Ababa between January and July 1984 it was noted that overall hospital infection in OBGY patient was $17 \%$ with wound infection. ${ }^{4}$ Shahid Arshad Meo reported an overall SSI of $1.91 \%$ study conducted at Feelege-Hiloot referral hospital, Ethiopa. ${ }^{2}$ While Jahanara et al reported incidence of $7.47 \%$ at Dhaka. ${ }^{5}$ our study shows the incidence of $8.33 \%$.

Melaku in 2012 reported that after comparing with gram positive and gram negative growth most common organism was Escherschia coli, Klebsiella and Pseudomonas followed by Staphylocoocus aureus which exactly matched with the present study. ${ }^{6}$ Several studies have reported an increasing role of Gramnegative organisms in hospital infections as opposed to the predominance of Staphylococcus aureus in the past. These groups of organisms tend to be endemic in hospital environment, being easily transferred from object to object, they also tend to be resistant to common disinfectants and antiseptics and are difficult to eradicate in the long term playing a great role in the many hospital acquired infections. ${ }^{7}$

The most common organism isolated from wound infection in our study is Gram negative Klebsiella and Pseudomonas i.e. $30.76 \%$ followed by Gram positive Staphylococcus aureus i.e $15.38 \%$ and then by Actinobacter, Non fermenter and mixed growth of Klebsiella and Pseudomonas 7.69\% each. In one study the most commonly reported pathogen was Staphylococcus aureus (40.4\%) of which $17.1 \%$ were methicillin-resistant. Other pathogens included anaerobic cocci (23.2\%), Enterobacteriaceae (13.3\%) and streptococci $(7.4 \%) .24 .2 \%$ were reported to be polymicrobial. $^{8}$

In a study done by Jahanara it was observed that incidence of wound infection was higher with emergency caesarean section than elective ones. ${ }^{6}$ whereas higher incidence of wound infection was seen in elective operation in present study than the emergency operation. Patients with anaemia were seen to be more prone to SSI. Anaemia diminishes resistance to infection and is frequently associated with puerperal sepsis. Preoperative anaemia is an important predictor of infection and has been proved by several other studies, ${ }^{9,10}$ Jahanara reported a strong relationship between anaemia and wound infection..$^{5}$ In our study also, anaemia was found to be significantly associated with SSI.

Premature rupture of membranes is associated with the largest bacterial inoculum and liquor gets infected and infection supervenes. $39.2 \%$ of patients who had a premature rupture of membranes or prolonged rupture (for more than 24 hours before surgery) were subsequently infected. ${ }^{11}$ It was found to be a significant risk factor in the study as was reported by several other authors ${ }^{12,13}$ In our study $15.38 \%$ patients with PROM showed wound infection. Hansis M in 1996 found that diabetes is an important factor of post operative wound infection. ${ }^{14}$ In our study Diabetics were more susceptible to infection than non diabetics.

\section{Conclusion}

The study has given us the knowledge about the surgical site infection and incidence in obstetrics and Gynecology Department in our hospital. A total of 300 cases were examined for surgical site infections in Obstetrics and Gynecology department and the overall incidence of infection was $8.33 \%$. There was an increase in incidence of SSI in patient with pre mature rupture of membrane i.e. $15.38 \%$, anemic patients show more SSI than patient with normal haemoglobin. The commonest organism isolated was Klebsiella and Pseudomonas followed Staphylococcus aureus.

\section{References}

1. Horan TC, Gaynes RP, Martone WJ, Jarvis WR, Emori TG. CDC definitions of nosocomial surgical site infections, 1992: a modification of CDC definitions of surgical wound infections. Am J Infect Control. 1992;20:271-4.

2. Meo SA, Siddique S, Nawaz Q, Meo RA. Frequency and patient related risks for surgical site infection. PAFMJ 2011; 61(2).

3. Collee JG, Marr W. Culture of bacteria "in Mackie and Macarteny practical medical; Microbiology, J.G. College, B.P. Marmion, G.F. Andrew and A. summons. Es 1996, PP 113-129, Churchill Livingstone Elesiver $14^{\text {th }}$ edition. 
4. Gedebou M, Habte-Gabr E, Kronvall G, Yoseph S. Hospital acquired infection among obstetrics and gynecological patient at Tikur Anbessa hospital, Addis Ababa. J Hosp Inf. 1988;11(1):50-59.

5. Rahman J, Sultana N, Hasan M, Begum HA. Factors of Post-operative wound infection in abdominal surgeries of Obstetrics and Gynaecology department. J Dhaka National Med Coll Hos. 2011;18(1):39-42.

6. Melaku S, Gebre- Selassie S, Damtie M, Alamrew K. Hospital acquired infection among surgical, gynaceology and obstetrics patients in felege-Hiwot referral hospital, Bahir dar, Northwest Ethopia. Ethiop Med J. 2012;50(2):135-144.

7. Makanjuola OB, Olowe OA, Adeyankinnu AF. Bacterial agents of surgical site infections in South-Western Nigeria. Am J Biomed Sci. 2013;5(4):217-225.

8. Wloch C, Wilson J, Lamagni T, Harrington P, Charlett A, Sheridan E. Risk factors for surgical site infection following caesarean section in England: results from a multicentre cohort study. BJOG. 2012;119(11):1324-33.

9. Zhou M, Chen L. Study of high-risk factors of surgical site infection after caesarean section. Di Jun Yi Da Xue Хие Bao. 2005;25(8):1075-1078.
10. Waisbren E, Rosen H, Eriksson E, Bader AM, Lipsitz SR, Rogers Jr. SO. Percent body fat and prediction of surgical site infection. J Am Coll Surg. 2010;210(4):381-9.

11. De D, Saxena S, Mehta G, Yadav R, Dutta R. Risk factor analysis and microbial etiology of surgical site infections following lower segment caesarean section. International Journal of Antibiotics. 2013;2013:283025.

12. Moir-Bussy BR. Hutton RM, Thompson JR. Wound infection after caesarean section. Journal of Hospital Infection. 1984;5(4):359-370.

13. Killian CA, Graffunder EM, Vinciguerra TJ, Venezia RA. Risk factors for surgical-site infections following caesarean section. Infection Control and Hospital Epidemiology. 2001;22(10):613-17.

14. Hansis M. Pathophysiology of Infection. Injury. 1996;27(3):SC5-8. 